\title{
LATTICE VIBRATIONS AND DISORDER IN CRYSTALLINE BENZOXAZOLES UNDERGOING EXCITED STATE INTRAMOLECULAR PROTON TRANSFER: DFTB MODELING
}

\author{
Y. Syetov* \\ Oles Honchar Dnipro National University, Dnipro, Ukraine \\ *e-mail:setov2003@yahoo.com
}

\begin{abstract}
Structure and crystal lattice vibrations are calculated for 2-(2'-hydroxyphenyl)bezoxazole and bis(2,5-benzoxazolyl)hydroquinone by density functional based tight-binding methods. Despite lowering of the molecular symmetry, structure parameters of the molecules in crystal and forms of the internal vibrations are similar to those of isolated molecules. Weak interaction between the molecules in the molecular crystals leads to appearance of the external vibrations, splitting and mixing of the vibrations of the isolated molecules into internal crystal vibrations. External and internal vibrations are not separated well; contribution of the translations and librations is noticeable in the region below $150 \mathrm{~cm}^{-1}$. The magnitude of the splitting does not exceed $4 \mathrm{~cm}^{-1}$ for the most vibrations. The internal vibrations that correspond to the out-of plane molecular vibrations demonstrate larger molecule-to-crystal frequency shift than in-plane modes, mostly to higher frequencies, whereas the modes involving torsion motion of the $\mathrm{OH}$ bond are shifted toward lower frequencies. Mixing occurs for the molecular vibrations with frequencies that are different by less than $16 \mathrm{~cm}^{-1}$. Calculations performed for model molecular clusters show that the defect caused by different molecule orientation has lower energy than the defect related to the formation of rotamers.
\end{abstract}

Keywords: molecular crystals, excited state proton transfer, vibrations, disorder, density-functional based tight-binding method.

Received 09.09.2018; Received in revised form 05.10.2018; Accepted 12.11.2018

\section{Introduction}

Photoinduced excited state intramolecular proton transfer (ESIPT) has attracted attention of researchers for several decades as an ultrafast photochemical reaction and a process that could be utilized for some applications including laser dyes and electroluminescent materials [1]. It has been found that the fluorescence spectra of some substances with ESIPT are dependent on polymorph modifications of the crystals while restriction of the large-amplitude motions of the molecules in the excited states by the crystal packing leads to the increase of the quantum yield in comparison with the liquid solution [2]. 2-(2'-hydroxyphenyl)benzoxazole (HBO) and 2,5-bis(2-benzoxazolyl)hydroquinone (BBHQ) are benzoxazoles that undergo ESIPT, both compounds demonstrate luminescence in the solid state [3, 4]. Molecules of HBO and BBHQ might exist in several intramolecularly Hbonded rotameric enol forms which differ by orientation of the benzoxazole moieties (Fig. 1).
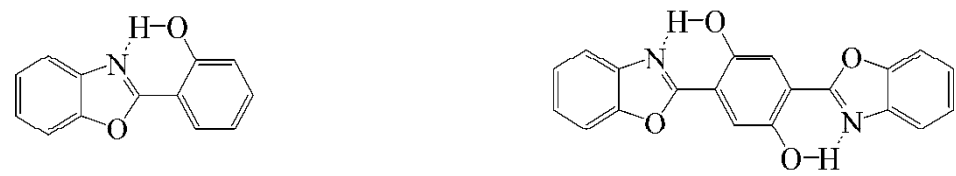

I

I<smiles>Oc1ccccc1-c1nc2ccccc2o1</smiles>

II

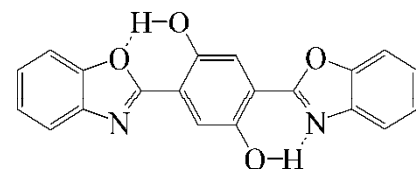

II

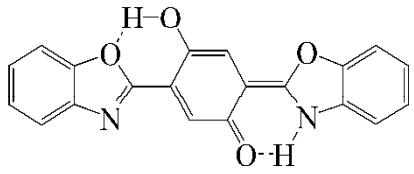

III

Fig. 1. Rotameric structures of molecules of HBO (a) and BBHQ (b). 
Only the forms with the $\mathrm{OH} . . . \mathrm{N}$ hydrogen bonds (I - for HBO, I and II - for BBHQ) are able to demonstrate ESIPT and fluorescence with anomalously large Stokes shift. Conformational disorder (approximately 1:1 ratio of structures I and II) has been determined by the X-ray study of HBO [5], however, only the fluorescence band with a large Stokes shift was found for crystalline HBO [6]. Recent X-ray measurements on the crystals of BBHQ were interpreted as existence of a kind of orientational disorder of structures I, but it was noted that the data allow interpreting the diffraction pattern as the presence of rotamers III, thus, existence of rotamer disorder [7].

The comparison of the IR spectra of crystalline powders of HBO and BBHQ in the region of intramolecular vibrations with the calculated spectra for different rotamers shows that the spectra of structures I demonstrate good correspondence with the experiment while the calculated bands originated from the vibrations of structures with $\mathrm{OH}$... O hydrogen bond are not found in the measured spectra. An IR spectrum of the related compound DBP which has non-hydrogen-bonded benzoxazole moiety comprises the bands that can be assigned to the structures with different orientation of the non-hydrogen-bonded benzoxazole moiety [8]. In the present paper we describe results of theoretical calculation of structure and vibrations of a crystal lattice of $\mathrm{HBO}$ and BBHQ.

\section{Calculations details}

Structure and vibrational modes of crystals HBO and BBHQ were calculated by density functional based tight-binding (DFTB) method with the third order correction and van der Waals interaction correction implemented in the DFTB + program package $[9,10]$. DFTB is an approximate quantum chemical method based on a second-order expansion of the KohnSham total energy in density functional theory (DFT) with respect to charge density fluctuations. DFTB is much faster than DFT and gives the possibility to calculate properties of molecules, clusters, and periodic structures comprising relatively large number of atoms. The van der Waals correction was done using the Lennard-Jones potential with the UFF parameters [11]. A $3 \times 3 \times 3$ supercell folding was used for the Brillouin zone sampling. The structure and phonon calculations were performed without disorder for the Brillouin zone center under the quasi-harmonic approximation that assumes the cell parameters fixed to the values taken from the X-ray data [5,7]. The DFT and DFTB calculations for isolated molecules were also performed with the GAMESS program suit $[12,13]$ using the B3LYP functional and 6-31G(d,p) basis set in the case of the DFT method. Vibrations of isolated molecules were calculated in harmonic approximation. Intensity of the infrared bands for the crystals was evaluated within the oriented gas model using nuclear derivatives of the dipole moments for the isolated molecule obtained by the DFTB calculations. In the oriented gas approximation the influence of intermolecular interactions on the optical properties of the molecules and the difference between macroscopic and local field are neglected [14].

\section{Results and discussion}

According to the X-ray data [5, 7], crystal of HBO belongs to orthorhombic structure class Pna2 ${ }_{1}$, BBHQ demonstrates monoclinic $\left(\mathrm{P} 2_{1}\right)$ crystal lattice with unit cell angles being close to $90^{\circ}$ (Fig. 2). An isolated molecule of $\mathrm{HBO}$ possesses $\mathrm{C}_{\mathrm{s}}$ symmetry and BBHQ $-\mathrm{C}_{2 \mathrm{~h}}$ symmetry. Molecules of $\mathrm{HBO}$ and BBHQ occupy general positions in the unit cells so that the cite symmetry group is $\mathrm{C}_{1}$ for both compounds and any molecular vibration can be related to any irreducible representation of crystal vibrations. The calculations show that a weak interaction between molecules in the crystals does not distort the structure significantly; the bond lengths differ only by less than $0.01 \AA$, valence angles - by the order of $0.1^{\circ}$. The molecules remain planar in crystals, the dihedral angles between benzoxazole and phenol (or 
hydroquinone) moieties are less than $3^{\circ}$. The fact that the structure parameters are close for isolated molecule and molecular units in the crystal allows decomposing the fundamental modes of the crystal lattice into the normal modes of an isolated molecule (including rotational and translational degrees of freedom) and normal coordinates of the free molecule can be considered as internal coordinates for the vibrations of the molecular units [14]. Coincidence of centers of mass and principal axes of inertia is assumed for the decomposition.

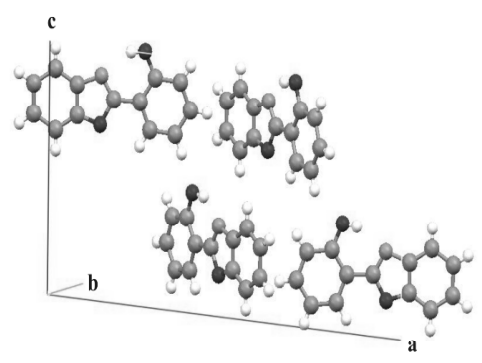

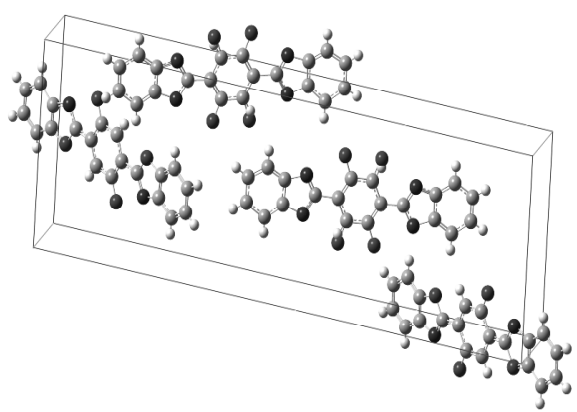

b

Fig. 2. Unit cells of crystals of $\mathrm{HBO}$ (a) and BBHQ (b) according to the X-ray measurements [5, 7]. For HBO unit cell only rotamers with an $\mathrm{OH}$.... hydrogen bond are shown, for BBHQ the orientational disorder is presented (coordinates of hydrogen atoms of the hydroxyl groups have not been reported for BBHQ).

The energy of defects caused by reorientation of the molecule in the lattice or formation of a rotamer is evaluated by calculations for the model clusters extracted from the optimized structure of fully ordered crystal. The cluster includes the molecule under consideration and the nearest neighbors. The positions of the atoms constituting the central molecule are optimized regarding the energy minimum. The coordinates of the atoms constituting the neighboring molecules are kept constant.

The energy of structure II of HBO is calculated by DFTB method to be $0.13 \mathrm{eV}$ larger than the energy of structure I (the DFT value is $0.27 \mathrm{eV}$ ). In the case of the model cluster, reorientation of the molecule that has structure I leads to the increase of the energy of the cluster by about $0.02 \mathrm{eV}$, while formation of the structure II leads to the increase of the energy by $0.15 \mathrm{eV}$. The last value demonstrates that the structure II is less stable in the cluster than in vacuum in comparison with structure I. Thus, reorientation of the molecule is more preferable energetically than formation of structure I. Estimation of the equilibrium concentration of the reoriented molecules according to the Boltzmann distribution gives 0.32. For BBHQ reorientation of the molecule increases energy of the cluster by much more large value of $0.55 \mathrm{eV}$ but the formation of the structure III to match the structure reported in [7] requires $0.75 \mathrm{eV}$. The energy difference between structures III and I for isolated molecule of BBHQ is calculated to be $0.26 \mathrm{eV}$ (DFT value is $0.53 \mathrm{eV}$ ). The calculations show that reorientation is preferable but the values for energy are too high (equilibrium concentration is about $10^{-10}$ ) and caused by decreases of the distance between the hydrogen atom of the hydroxyl group and the hydrogen atom of the aromatic ring of the neighboring molecules.

Although the DFTB calculations for a crystal have been performed without using the symmetry, the obtained structure and phonon eigenvectors approximately satisfy the symmetry relations. A noticeable contribution (more than 0.1 for one molecule in the decomposition) of the external coordinates is found for vibrations with the frequencies below 
$150 \mathrm{~cm}^{-1}$ in the case of HBO and $140 \mathrm{~cm}^{-1}$ for BBHQ. The value found for HBO corresponds to the region of intense bands observed in the Raman spectrum [15]. In the region of internal vibrations the most vibrations can be considered as molecular vibrations split into 4 Davydov components, i.e. the decomposition into the free molecule modes comprises one dominant term. The magnitude of splitting does not exceed $4 \mathrm{~cm}^{-1}$ for the most vibrations, only for the vibration with frequency $151 \mathrm{~cm}^{-1}$ the splitting is found to be $7 \mathrm{~cm}^{-1}, 237 \mathrm{~cm}^{-1}-5 \mathrm{~cm}^{-1}, 1051$ $-12 \mathrm{~cm}^{-1}$ for HBO; $408 \mathrm{~cm}^{-1}-6 \mathrm{~cm}^{-1} 1305 \mathrm{~cm}^{-1}-10 \mathrm{~cm}^{-1}$ for BBHQ. Beyond splitting the vibration frequencies in the crystals are shifted in comparison with the frequencies of the molecule vibrations. The shift estimation as a difference of the average frequency of the Davydov quartet and frequency of the molecule vibration is more pronounced for out-ofplane vibrations and reaches $32 \mathrm{~cm}^{-1}$ toward the higher frequencies for the mode $151 \mathrm{~cm}^{-1}$ $(\rho($ phO-10b) $+\rho($ ph-10a) according to Varsanyi's notation [15]) of the molecule of HBO. The torsion vibration involving $\mathrm{OH}$ bond undergoes a shift of $26 \mathrm{~cm}^{-1}$ toward lower frequencies. In the crystal of BBHQ the largest shift of $34 \mathrm{~cm}^{-1}$ toward higher frequencies is found for outof-plane vibration $205 \mathrm{~cm}^{-1}$ ( $\rho(\mathrm{ph}-10 \mathrm{~b})$ of benzoxazole moieties). The $\mathrm{OH}$ torsion vibrations 555 and $558 \mathrm{~cm}^{-1}$ exhibit shift to the lower frequency of about $26 \mathrm{~cm}^{-1}$, mixing of the vibrations (lowering the symmetry), and mixing with the in-plane vibration $530 \mathrm{~cm}^{-1}$. The shift of about $23 \mathrm{~cm}^{-1}$ and mixing is found for $\mathrm{OH}$ stretching vibrations 3434 and $3438 \mathrm{~cm}^{-1}$ as well. A number of the modes of crystals demonstrate that the deviations of an atom upon vibrations can be decomposed into several molecular modes with close frequencies. Molecular modes of HBO with calculated frequencies $458\left(\mathrm{~A}^{\prime \prime}\right)$ and $469\left(\mathrm{~A}^{\prime}\right), 742\left(\mathrm{~A}^{\prime \prime}\right)$ and $751\left(\mathrm{~A}^{\prime \prime}\right), 865\left(\mathrm{~A}^{\prime \prime}\right)$ and $876\left(\mathrm{~A}^{\prime}\right), 894\left(\mathrm{~A}^{\prime \prime}\right)$ and $900\left(\mathrm{~A}^{\prime \prime}\right), 1131\left(\mathrm{~A}^{\prime}\right)$ and $1143\left(\mathrm{~A}^{\prime}\right), 1164\left(\mathrm{~A}^{\prime}\right)$ and $1169\left(\mathrm{~A}^{\prime}\right), 1281\left(\mathrm{~A}^{\prime}\right)$ and $1290\left(\mathrm{~A}^{\prime}\right), 3002\left(\mathrm{~A}^{\prime}\right)$ and $3007\left(\mathrm{~A}^{\prime}\right), 3018\left(\mathrm{~A}^{\prime}\right)$ and $3024 \mathrm{~cm}^{-1}$ (A') are found to have comparable contributions to corresponding crystal modes. Noticeable lowering of the symmetry, thus, occurs for 4 molecular modes. In BBHQ the mixing is found for vibrations $175\left(\mathrm{~A}_{\mathrm{u}}\right)$ and $185\left(\mathrm{~A}_{\mathrm{g}}\right), 307\left(\mathrm{~A}_{\mathrm{u}}\right)$ and $322\left(\mathrm{~B}_{\mathrm{u}}\right), 451\left(\mathrm{~A}_{\mathrm{u}}\right), 459\left(\mathrm{~B}_{\mathrm{g}}\right)$ and $460\left(\mathrm{~B}_{\mathrm{u}}\right)$, $530\left(\mathrm{~B}_{\mathrm{u}}\right), 555\left(\mathrm{~B}_{\mathrm{g}}\right)$ and $558\left(\mathrm{~A}_{\mathrm{u}}\right), 561\left(\mathrm{~A}_{\mathrm{u}}\right), 562\left(\mathrm{~B}_{\mathrm{g}}\right)$ and $567\left(\mathrm{~A}_{\mathrm{g}}\right), 609\left(\mathrm{~B}_{\mathrm{u}}\right)$ and $609\left(\mathrm{~A}_{\mathrm{u}}\right), 723$ $\left(\mathrm{A}_{\mathrm{g}}\right), 724\left(\mathrm{~B}_{\mathrm{g}}\right)$ and $724\left(\mathrm{~A}_{\mathrm{u}}\right), 745\left(\mathrm{~B}_{\mathrm{g}}\right)$ and $746\left(\mathrm{~A}_{\mathrm{u}}\right), 811\left(\mathrm{~B}_{\mathrm{g}}\right), 813\left(\mathrm{~A}_{\mathrm{u}}\right), 817\left(\mathrm{~B}_{\mathrm{g}}\right)$ and 817 $\left(\mathrm{A}_{\mathrm{u}}\right), 866\left(\mathrm{~B}_{\mathrm{g}}\right), 866\left(\mathrm{~A}_{\mathrm{u}}\right)$ and $874\left(\mathrm{~B}_{\mathrm{u}}\right), 895\left(\mathrm{~B}_{\mathrm{g}}\right)$ and $895\left(\mathrm{~A}_{\mathrm{u}}\right), 1051\left(\mathrm{~A}_{\mathrm{g}}\right)$ and $1052\left(\mathrm{~B}_{\mathrm{u}}\right), 1163$ $\left(\mathrm{B}_{\mathrm{u}}\right)$ and $1164\left(\mathrm{~A}_{\mathrm{g}}\right), 1287$ and $1287\left(\mathrm{~A}_{\mathrm{g}}\right), 1455\left(\mathrm{~B}_{\mathrm{u}}\right)$ and $1456\left(\mathrm{~A}_{\mathrm{g}}\right), 1518\left(\mathrm{~B}_{\mathrm{u}}\right)$ and $1518\left(\mathrm{~A}_{\mathrm{g}}\right)$, $3002\left(\mathrm{~B}_{\mathrm{u}}\right)$ and $3002\left(\mathrm{~A}_{\mathrm{g}}\right), 3009\left(\mathrm{~B}_{\mathrm{u}}\right)$ and $3009\left(\mathrm{~A}_{\mathrm{g}}\right), 3018\left(\mathrm{~A}_{\mathrm{g}}\right)$ and $3018\left(\mathrm{~B}_{\mathrm{u}}\right), 3023\left(\mathrm{~B}_{\mathrm{u}}\right)$ and $3023\left(\mathrm{~A}_{\mathrm{g}}\right), 3435\left(\mathrm{~B}_{\mathrm{u}}\right)$ and $3438 \mathrm{~cm}^{-1}\left(\mathrm{~A}_{\mathrm{g}}\right)$. Nevertheless, upon the mixing, some crystal vibrations related to these molecular modes retain one dominant component in the decomposition into molecular modes. The IR spectra of free molecules and crystalline powders calculated by DFTB method using the oriented gas model are shown in Fig. 3. The IR bands were modeled by Gaussian lineshape with full width at half maximum of $4.0 \mathrm{~cm}^{-1}$. In Fig. 3 the IR spectra calculated by DFT method for free molecules are presented for comparison. IR spectra calculated by DFT demonstrate good agreement with the experimental spectra of powders in the region $400-4000 \mathrm{~cm}^{-1}$ [8]. Due to small magnitude of splitting, for the most vibrations the splitting does not exceed the width of bands, the intensity of IR bands for crystalline powder is the sum of the intensity of their components and does not differ significantly from the intensity of IR bands of free molecule. The spectrum difference is caused by frequency shift and mixing of the molecular modes, the letter leads to IR intensity redistribution. The $\mathrm{OH}$ stretching vibrations in the crystal of BBHQ are expected to demonstrate two bands instead of one in the molecule (Fig. 3) but in the experimental spectrum the corresponding bands are wide and structured due to the anharmonic interaction with low-frequency vibrations that change the distance between atoms $\mathrm{N}$ and $\mathrm{O}$ involved in the hydrogen bonds [8]. 


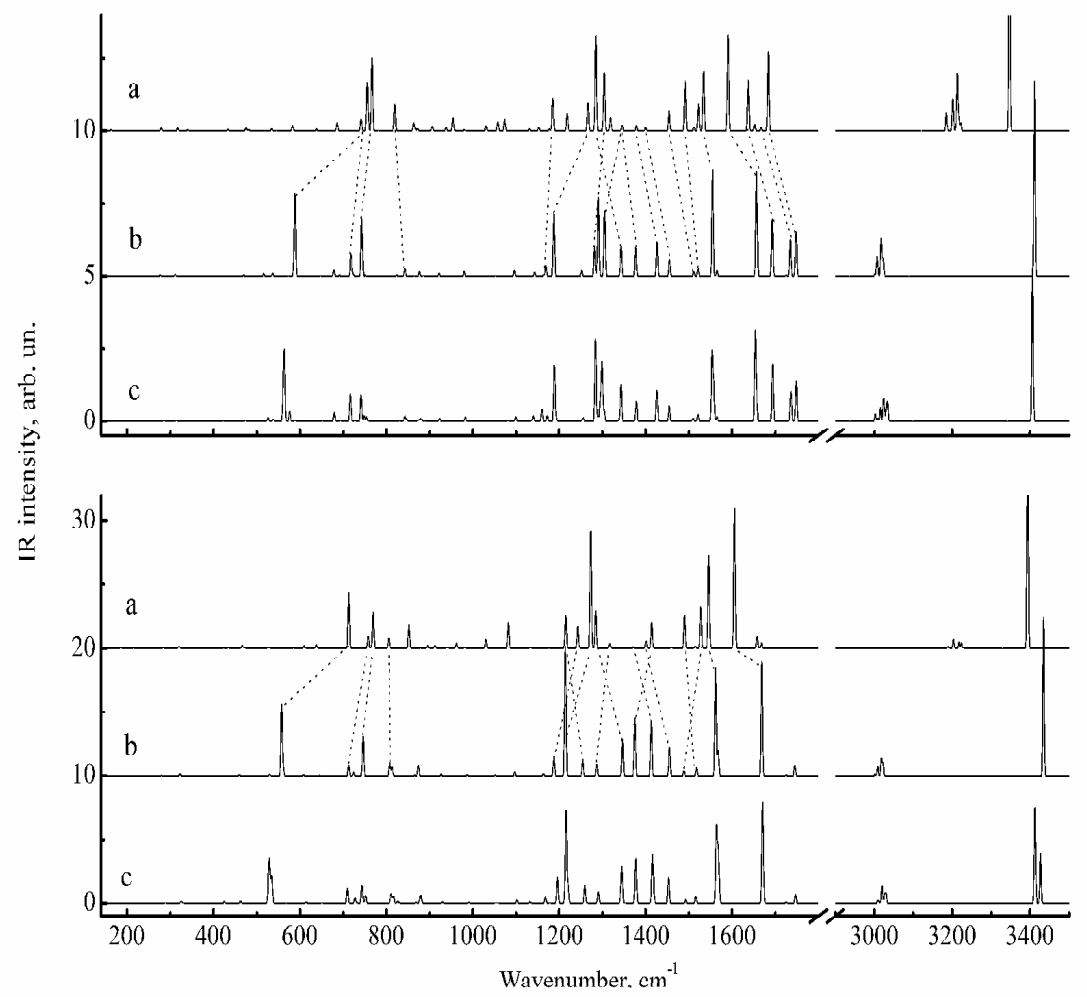

Fig. 4. Calculated IR spectra of HBO (upper graph) and BBHQ (lower graph) in the region of internal vibrations: a - results of DFT calculations for a free molecule; $b$ - results of DFTB calculations for a free molecule; $c$ - results of DFTB calculations for an ordered crystal within the oriented gas model. Dashed lines denote the maximum correspondence between forms of the selected vibrations.

Comparison with the frequencies and forms of vibrations calculated by the DFT methods for free molecules demonstrate that DFTB method yields reasonable frequencies within $30 \mathrm{~cm}^{-1}$ and similar forms only in the region below $600 \mathrm{~cm}^{-1}$, substantially underestimates the frequency of the $\mathrm{OH}$ torsion vibrations (more than $150 \mathrm{~cm}^{-1}$ ) and exhibit large non-systematic deviations for the vibrations in the regions $1290-1380 \mathrm{~cm}^{-1}$ and $1500-$ $1570 \mathrm{~cm}^{-1}$, and for BBHQ in the region $1180-1570 \mathrm{~cm}^{-1}$ (Fig. 3, dashed lines).

\section{Conclusions}

The calculations of structure and vibrations of the crystal lattice of benzoxazoles HBO and BBHQ by means of densityfunctional based tight-binding methods show that the interaction of the molecules in the crystals causes the appearance of external vibrations in the range below $150 \mathrm{~cm}^{-1}$. The internal vibrations of the lattice retain similarity to the vibrational modes of free molecules, deviation of vibration forms in a crystal from molecular vibrations occurs in the case of close frequencies of several molecular modes. Structure parameters of the molecules in crystals are close to the parameters of free molecules, the reorientation of molecules in the lattice requires less energy than formation of rotamers.

\section{References}

1. Hsieh, C-C. Organic Dyes with Excited-State Transformations (Electron, Charge, and Proton Transfers) / C-C. Hsieh, M-L. Ho, P-T. Chou // Advanced Fluorescence Reporters 
in Chemistry and Biology I: Fundamentals and Molecular Design. Ed. by Demchenko A. P. Springer Ser. Fluoresc. - 2010. - Vol. 8. - P. 225-266.

2. Konoshima H. Excited-state intramolecular proton transfer and charge transfer in 2(2'-hydroxyphenyl)benzimidazole crystals studied by polymorphs-selected electronic spectroscopy / H. Konoshima, S. Nagao, I. Kiyota, K. Amimoto, N. Yamamoto, M. Sekine, M. Nakata, K. Furukawaa, H. Sekiya // Phys. Chem. Chem. Phys. - 2012. - Vol. 14. - P. 16448-16457.

3. Williams D. L. Intramolecular proton transfer reactions in excited fluorescent compounds / D. L. Williams, A. Heller // J. Phys. Chem. - 1970. - Vol. 24. - P. 4473-4480.

4. Orlando C. M. Red- and near-infrared luminescent benzazole derivatives / C. M. Orlando, J. G. Wirth, D. R. Heath // Chem. Comm. - 1971. - Vol. 48. - P. 1551-1552.

5. Tong Y.-P. 2-(2-Hydroxyphenyl)-1,3-benzoxazole / Y.-P. Tong // Acta Cryst. E. 2005. - Vol. 61. - P. o3076 - o3078.

6. Syetov, Y. Luminescence spectrum of 2-(2'-hydroxyphenyl)benzoxazole in the solid sate / Y. Syetov // Ukr. J. Phys. Opt. - 2013. - Vol. 14, № 1. - P. 1-5.

7. Vdovin A. 2,5-bis(2'-benzoxazolyl)hydroquinone (BBHQ), a dually fluorescent ESIPT system revisited: XRD analysis and supersonic jet studies of deuterated species / A. Vdovin, E. Karpiuk J. Lipkowski, A.Listkowski, M. Kijak, A. Grabowska, J.Sepioł // J. Mol. Struct. - 2018. - Vol. 1171. - P. 843-849.

8. Syetov Y. Infrared spectra of benzoxazoles exhibiting excited state proton transfer / Y. Syetov, A. Vdovin // Vib. Spectrosc. - 2010. Vol. 53. - P. 146-150.

9. Aradi B. DFTB+, a Sparse Matrix-Based Implementation of the DFTB Method / B. Aradi, B. Hourahine, Th. Frauenheim // J. Phys. Chem. A - Vol. 111(26). - 2007. - P. 56785684.

10. Gaus M. DFTB3: Extension of the Self-Consistent-Charge Density-Functional Tight-Binding Method (SCC-DFTB) / M. Gaus, Q. Cui, M. Elstner // J. of Chem. Theory Comput. - 2011. - Vol. 7. - P. 931-948.

11. Rappe A. K. Uff, a full periodic table force field for molecular mechanics and molecular dynamics simulations / A. K. Rappe, C. J. Casewit, K. S. Colwell, W. A. Goddard III, W. M. Skiff // J. Am. Chem. Soc. - 1992. - Vol. 114. - P. 10024-10035.

12. Schmidt M. W. General Atomic and Molecular Electronic Structure System / M. W. Schmidt, K. K. Baldridge, J. A. Boatz, S. T. Elbert, M. S. Gordon, J. H. Jensen, S. Koseki, N. Matsunaga, K. A. Nguyen, S. J. Su, T. L. Windus, M. Dupuis, J. A. Montgomery // J. Comput. Chem. - 1993. - Vol. 14. - P. 1347-1363.

13. Gordon M. C. Advances in electronic structure theory: GAMESS a decade later / M. C. Gordon, M. W. Schmidt // Chapter 41 in Theory and Applications of Computational Chemistry, the first forty years / Ed. by Dykstra C. E., Frenking G., Kim K. S., Scuseria G. E. - Amsterdam: Elsevier. - 2005. - P. 1167-1189.

14. Zhizhin G. N. Optical Spectra and Lattice Dynamics of Molecular Crystals / G. N. Zhizhin, E. I. Mukhtarov. - 1st edition. - Amsterdam: Elsevier Science, 1995. - 446 p.

15. Syetov Y. Low-frequency Raman spectrum of crystalline 2-(2'hydroxyphenyl)benzoxazole and density-functional based tight-binding phonon calculations / Y. Syetov // Ukr. J. Phys. Opt. - 2017. - Vol. 18, № 2. - P. 67-76. 